\title{
Ingrid Stober und Kurt Bucher: Geothermie
}

\author{
Springer Geology, Spinger-Verlag Berlin Heidelberg 2012, \\ ISBN 978-642-24330-1, 287 Seiten
}

\section{Ladislaus Rybach}

Online publiziert: 07. November 2012

(C) Springer-Verlag Berlin Heidelberg 2012

Das Buch beschreibt die vielfältigen Nutzungsmöglichkeiten geothermischer Energieressourcen. Bis zum Bau und Betrieb von Geothermie-Anlagen führt ein langer Weg. Es braucht dabei viele Weggefährten: Spezialisten aus zahlreichen Disziplinen. Strukturgeologen und Hydrogeologen, Geophysiker und Geochemiker, Bohr- und Reservoir-Ingenieure, Anlageplaner und Ökonomen, aber auch Umwelt-Beamte, Juristen und Versicherungsfachleute müssen zielgerichtet und effizient zusammenarbeiten. Dies um die speziellen Fragestellungen zu verstehen und dadurch zu möglichen Antworten zu gelangen. Hierzu bietet dieses Buch eine willkommene Hilfe, denn darin findet der Leser nicht nur Einblicke, sondern gewinnt auch Fachkenntnisse.

Das Buch ist thematisch gut strukturiert und reich illustriert. Besonders eindrücklich sind die vielen praxisnahen Fotos (alle von Ingrid Stober aufgenommen). Die Einleitung positioniert die Geothermie durch Beschreibung ihrer mannigfachen Vorteile als eine wichtige Komponente von künftigen, nachhaltigen Energieversorgungssystemen. Nach einer Einführung in die Grundlagen der Geothermie werden die Geothermie-Ressourcen und ihre Nutzungsmöglichkeiten vorgestellt. Ein Schwergewicht bilden die Technologien mittels Erdwärmepumpen und geothermischer Brunnen- sowie Doubletten-Anlagen. Die geothermische Zukunftsvision „Enhanced Geothermal Systems“ (EGS) und ihre noch ungelösten Fragen sind genauso dargestellt wie die etablierte Bohrtechnik für Tiefbohrungen. Auch werden geophysikalische Verfahren, hydraulische Tests und hydrochemische Untersuchungen beschrieben. Potentielle
Umweltauswirkungen der Tiefen Geothermie (wichtig für die soziale Akzeptanz) werden auch dargestellt.

Einige kleinere Unstimmigkeiten könnten bei einer Neuauflage (oder bei einer englischen Version) behoben werden:

- heute stehen wesentlich neuere statistische Daten zur Verfügung (z. B. bei REN21) als jene auf S. 2

- die Aussage, dass die Wärme aus dem radioaktiven Zerfall in der Erdkruste kontinuierlich neu gebildet wird (S. 9), stimmt nur für Kontinente

- Gleichung (1.5.a, S. 13) stimmt so nicht

- im Text sollte einheitlich der Begriff „Erdwärmesonde“ verwendet werden

- für das Diagramm in Abb. 8.8 (S. 143) soll der Bohrlochdurchmesser angegeben werden

- bei den Ausfällungen u. a. in Rohrleitungen (S. 144) soll auf den erfolgreichen Einsatz von Inhibitoren hingewiesen werden

- bei Abb. 13.10 (S. 242) fehlen die Kurvenparameter

Erstmals wird die Geothermie in diesem Buch in ihrer vollen Breite abgedeckt und übersichtlich dargestellt. Neben Fachleuten finden hier auch Studierende nützliche Informationen, denn über Spezialgebiete wie z. B. numerische Modellierung sind weiterführende Literaturangaben aufgeführt. Ein sehr empfehlenswertes Buch also!

Ladislaus Rybach, Prof.em. Dr.Ing. Dr.h.c. Institut für Geophysik, ETH Zürich, Schweiz

L. Rybach $(\triangle)$

Institute of Geophysics, ETH Zentrum,

8092 Zürich, Schweiz

E-Mail: rybach@ig.erdw.ethz.ch 